Obuchowska Karolina, Obuchowska Aleksandra, Standyło Arkadiusz, Wójcik Justyna, Ozga Alicja. The relationship between the occurrence of psoriasis and depression. Journal of Education, Health and Sport. 2020;10(9):403-406. eISSN 2391-8306. DOI http://dx.doi.org/10.12775/JEHS.2020.10.09.047

https://apcz.umk.pl/czasopisma/index.php/JEHS/article/view/JEHS.2020.10.09.047

https://zenodo.org/record/4035713

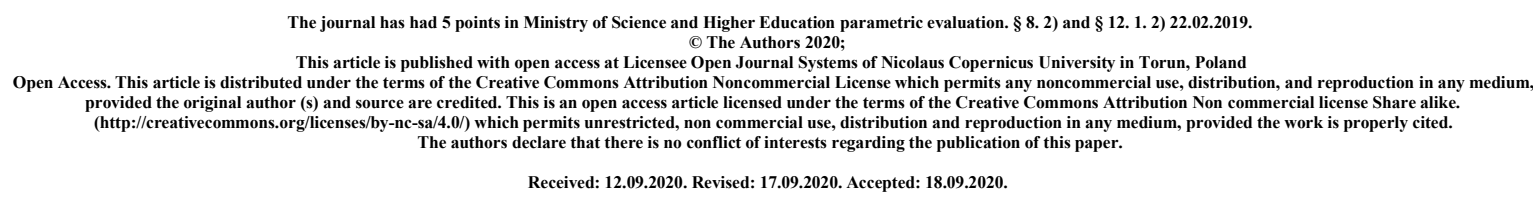

\title{
The relationship between the occurrence of psoriasis and depression
}

\author{
Karolina Obuchowska $^{1 \mathrm{a}}$, Aleksandra Obuchowska ${ }^{1 \mathrm{~b}}$, Arkadiusz Standyło ${ }^{1 \mathrm{c}}$, \\ Justyna Wójcik' ${ }^{1 \mathrm{~d}}$, Alicja Ozga ${ }^{1 \mathrm{e}}$
}

\author{
${ }^{1}$ Student Scientific Group at the Medical University of Lublin, Aleje Racławickie Street 1, \\ 20-059 Lublin \\ a karolinaobuchowska99@gmail.com; https://orcid.org/0000-0003-4519-8236 \\ b aobuchowska12@gmail.com; https://orcid.org/0000-0003-0464-2695 \\ c a.standylo@gmail.com; https://orcid.org/0000-0002-5154-4759 \\ d justynawojcik455@gmail.com; https://orcid.org/0000-0001-7163-6784 \\ eaozga1@gmail.com; https://orcid.org/0000-0003-1291-905X
}

\section{Summary}

Psoriasis (skin psoriasis, $\mathrm{PsO}$ ) is a chronic auto-immune skin inflammatory condition. In about one-third of cases, the joints are affected (psoriatic arthritis, PsA). Both conditions, especially PsA, profoundly impact patients' health-related quality of life. This disease affects between $2 \%$ and $3 \%$ of the population. The onset occurs before the age of 40 as a sharply demarcated scaly, red skin lesions, most often on the elbows, knees, scalp, hands, feet and joints considering osteoarticular involvement. Patients are suffering from itching, irritations, stinging and pain. Etiology of this disease is multifactorial, with genetic and environmental involvement, psychological stress and depression being the key factors considering the onset, flare or therapeutic resistance of psoriatic disease. The purpose of this article is to review and analyze how depression and stress affect the life of patients with psoriasis and in which way prevention could play a role regarding the evolution of this disease. 
Proinflammatory cytokines such as interleukin (IL)-1 and IL-6 are elevated in both psoriasis and depression, indicating that the inflammatory process may be involved in the progression of both diseases. Psoriasis has long been recognized to be associated with potentially adverse effects on mental health. However, there have been relatively few studies evaluating psychological outcomes in patients with psoriasis.

Depression and stress decrease the quality of life, psoriasis being the last drop in the glass, with bimodal intensification of these pathologies. Quality of life in patients with psoriasis is directly associated with the severity of the disease, family status, work status and stigmatization.

Key words: psoriasis, depression, quality of life

\section{Introduction}

Psoriasis is a common chronic condition that affects $1-3 \%$ of general population and based on the daily consultations it is one of the most frequently presented skin disorders. Furthermore valuations suggests that $0.4-2.3 \%$ of the adult population have psoriasis but remain undiagnosed [1,2]. Psoriasis is also a genetic disease whose appearance is related to environmental factors such as family problems, poor standards of living, high-pressure situations [3]. This disease is characterized by appearance of red, scaly skin lesions. The most common are the hands, elbows, knees, feet, scalp and joints [2,4]. The most difficult ones to treat are lesions located on the hands or feet. Although no matter where the lesions are located, they induce dermatological symptoms such as pain, swelling and itching, which can have a significant effect on patient's everyday life [4].

\section{Purpose}

The purpose of this article is to review and analyze how depression and stress affect the life of patients with psoriasis.

\section{Description of the state of knowledge}

Clinically, the severity of psoriasis is described by the severity and magnitude of skin changes. The most common and credible instrument which allows to measure the severity of psoriasis is Psoriasis Area and Severity Index (PASI). PASI is based on the extension of area of the body which is covered by erythema and induration before and after treatment. PASIx is described by the $\mathrm{x} \%$ reduction compared to baseline. PASI score exceeding 12 is signifies as severe form of psoriasis, 7-12 as moderate and beneath 7 as mild [2,5]. From the point of view of the patient's the influence of this disease on their everyday mental, social and physical living is most important [5]. The skin lesions, caused by psoriasis are frequently very noticeable, which can lead to psychological problems such as stigmatization, anxiety and depression [6]. Considering the mental state and quality of life of psoriatic patients Dermatology Life Quality Index (DLQI) was created. It is In the form of questionnaire intended for patients aged over 16. DLQI contains of 10 questions, each one scoring from 0 to 3 and the maximum overall score of 30 . Moderate and severe psoriasis is correlated with high DLQI score, similarly stores of patients with depression and long-term stress. 
Although successful therapy results in decreasing of DLQI and PASI [3,4]. Psoriasis Life Stress Inventory (PLSI) allows to determine the level of stress perceived during everyday situations by people with psoriasis. The final version of the PLSI consists of 15 items about potentially stressful situations they had experienced over the previous month and rates the degree of stress associated with each item on a 4-point scale. It shows that the more intensive psoriasis is, the higher PLSI is rated [5,7]. Study on patients with psoriasis conducted by Akay shows that they have extremely high Beck Depression stores. It was associated with area of psoriatic lesions and depression score was higher than the non-psoriasis-afflicted person get [3]. Studies about pathophysiology of psoriasis show that this disease is due to the level of macrophages, $\mathrm{T}$ cells, and migration of dendric cells from dermis to epidermis. Those cells afterwards release inflammatory citokines such as TNF- $\alpha$, interleukins: 1-beta, 6,22 or interferon-y. Furthermore there is a defect in regulatory T cells and regulatory cytokine IL-10. These substances released by the cells lead to enlargement of inflammatory process and deepening of psoriasis. The level of proinflammatory cytokines is elevated in patients with depression compared to the health population, so it could lead to correlation between psoriasis and depression. Psoriasis, however, does not just affect the skin, as elevated cytokine levels are observed in the blood in patients with psoriasis. Notably, IL-6 can exert proinflammatory effects in a range of organ systems and has been implicated in a variety of diseases, including cancer, heart disease, depression, rheumatoid arthritis and psoriasis $[3,4,8]$.

\section{Summary}

In conclusion, patients with psoriasis are at increased risk for depression, anxiety and suicidality compared to the general population. Comorbid depression and psoriasis interrelate negatively, giving rise to a dangerous vicious circle. The impact of depression can be reduced by promptly diagnosing and treating depression while treating psoriasis. It is important to understand the impacts that psoriasis can have on quality of life, including physical and mental health. 


\section{References:}

1. Kurd SK, Troxel AB, Crits-Christoph P, Gelfand JM. The risk of depression, anxiety, and suicidality in patients with psoriasis: a population-based cohort study. Arch Dermatol. 2010;146(8):891-895. doi:10.1001/archdermatol.2010.186

2. Rigas HM, Bucur S, Ciurduc DM, Nita IE, Constantin MM. Psychological Stress and Depression in Psoriasis Patients - a Dermatologist's Perspective. Maedica (Buchar). 2019;14(3):287-291. doi:10.26574/maedica.2019.14.3.287

3. Tohid H, Aleem D, Jackson C. Major Depression and Psoriasis: A Psychodermatological Phenomenon [published correction appears in Skin Pharmacol Physiol. 2016;29(5):280]. Skin Pharmacol Physiol. 2016;29(4):220-230. doi:10.1159/000448122

4. Koo J, Marangell LB, Nakamura M, et al. Depression and suicidality in psoriasis: review of the literature including the cytokine theory of depression. J Eur Acad Dermatol Venereol. 2017;31(12):1999-2009. doi:10.1111/jdv.14460

5. Schmitt J, Ford DE. Understanding the relationship between objective disease severity, psoriatic symptoms, illness-related stress, health-related quality of life and depressive symptoms in patients with psoriasis - a structural equations modeling approach. Gen Hosp Psychiatry. 2007;29(2):134-140. doi:10.1016/j.genhosppsych.2006.12.004

6. Baker CS, Foley PA, Braue A. Psoriasis uncovered--measuring burden of disease impact in a survey of Australians with psoriasis. Australas J Dermatol. 2013;54 Suppl 1:1-6. doi:10.1111/ajd.12010

7. Gupta MA, Gupta AK. The Psoriasis Life Stress Inventory: a preliminary index of psoriasis-related stress. Acta Derm Venereol. 1995;75(3):240-243.

doi:10.2340/0001555575240243

8. González-Parra S, Daudén E. Psoriasis and Depression: The Role of Inflammation. Psoriasis y depresión: el papel de la inflamación. Actas Dermosifiliogr. 2019;110(1):12-19. doi:10.1016/j.ad.2018.05.009 